\title{
When the Heart Triggers the Esophagus: Esophageal Spasm after Electrical Cardioversion
}

\author{
$\underline{\text { Riccardo Manfredo Campaner }}{ }^{1}$, Marco Enzo Tau ${ }^{1}$, Francesca Ortelli ${ }^{1}$, Maria Luisa De Perna ${ }^{2}$, Tanja Fusi-Schmidhauser ${ }^{1}$ \\ ${ }^{1}$ Department of Internal Medicine, Ospedale Regionale di Lugano, Ente Ospedaliero Cantonale, Switzerland \\ ${ }^{2}$ Department of Cardiology, Ospedale Regionale di Lugano, Ente Ospedaliero Cantonale, Switzerland
}

Received: 05/11/2019

Accepted: $11 / 11 / 2019$

Published: 11/12/2019

\begin{abstract}
How to cite this article: Campaner RM, Tau ME, Ortelli F, De Perna ML, Fusi-Schmidhauser T. When the heart triggers the esophagus: esophageal spasm
\end{abstract} after electrical cardioversion. EJCRIM 2019;6: doi:10.12890/2019_001369.

Conflicts of Interests: The Authors declare that there are no competing interest

Acknowledgements: Riccardo Manfredo Campaner contributed to the conception and design of the study, and drafting of the manuscript; Marco Tau, Francesca Ortelli and Maria Luisa De Perna contributed to the conception and design of the study, and drafting of the manuscript; Tanja Fusi-Schmidhauser contributed to revision and gave approval of the final version of the manuscript.

This article is licensed under a Commons Attribution Non-Commercial 4.0 License

\section{ABSTRACT}

Introduction: "Esophageal spasm" is a generic term widely used to attribute unexplained non-cardiac chest pain and/or dysphagia to an esophageal motility disorder.

Patient and methods: The authors present the case of an 86-year-old male patient with complete dysphagia after an elective electrical cardioversion for atrial fibrillation. An upper endoscopy performed shortly after the onset of the clinical picture documented disordered esophageal contractions. The patient became asymptomatic within 12 hours of the administration of a spasmolytic therapy.

Results: To the best of our knowledge, this is the first report of esophageal spasm after an electrical cardioversion.

Discussion: The temporal correlation supports the explanation of a cause-effect relationship between the clinical presentation and the preceding procedure, thus providing elements for clinicians to recognize and treat this particular condition.

\section{LEARNING POINTS}

- This case report suggests that electrical cardioversion may induce clinically relevant esophageal spasms.

- When dealing with dysphagia after an electrical cardioversion, an empirical spasmolytic treatment can be considered, if not contraindicated.

\section{KEYWORDS}

Esophageal spasm, dysphagia, electrical cardioversion, atrial fibrillation

\section{INTRODUCTION}

Esophageal motility disorders are a heterogeneous family of disorders characterized by non-propulsive contractions, hyperdynamic contractions or high pressure of the lower esophageal sphincter. Presenting symptoms are more frequently chest pain and dysphagia ${ }^{[1]}$. Although diagnosis of this kind of motility disorder is established by manometric study according to the Chicago Classification, endoscopy may rarely reveal disordered esophageal contractions. The pathogenesis still remains uncertain but hyperexcitation and increased smooth muscle thickness are evoked. Therapeutic options are not standardized and include calcium channel blockers, botulinum toxin injection or surgical myotomy ${ }^{[2,3]}$. Until now, there have been no described cases of esophageal motility disorder that occurred after electrical cardioversion. 


\section{CASE DESCRIPTION}

We describe the case of an 86-year-old male patient, who was admitted to the internal medicine ward for the recurrence of a paroxysmal atrial fibrillation with rapid ventricular response. The previous medical history was characterized by a recent embolic stroke secondary to a novel diagnosed atrial fibrillation without residual neurological impairment. The patient was started on therapeutic anticoagulation therapy with apixaban $5 \mathrm{mg}$ twice a day, in association with pantoprazole. On that occasion, a speech therapist excluded deglutition impairment and dysphagia. In addition, the patient was known for essential hypertension and benign prostatic hyperplasia. He never suffered from gastro-o esophageal diseases or symptoms. Since a rate control strategy with a beta-blocker and digoxin was not effective and no reversible triggers for the atrial fibrillation recurrence were identified, a rhythm control approach was pursued. As the patient was regularly assuming anticoagulation therapy for more than 4 weeks, trans-esophageal echocardiography was not required before electrical cardioversion. The procedure was performed under procedural sedation and analgesia with, respectively, propofol and fentanyl. Defibrillator pads were placed in the anterior-posterior position, according to international guidelines. Electrical cardioversion entailed a first, ineffective, biphasic DC shock (120 joules), followed by an immediate second, effective, biphasic DC shock (120 joules), with sinus rhythm restoration. Several hours after the procedure, during a meal, the patient reported the onset of progressive complete dysphagia for both liquids and solids. The otorhinolaryngologist confirmed a regular deglutition. Therefore, the patient underwent an esophagogastroduodenoscopy (EGDS), which identified an endoscopic pattern suggestive of nutcracker esophagus along the more caudal $15 \mathrm{~cm}$, without mucosal lesion. The patient was treated with a spasmolytic agent (nifedipine $30 \mathrm{mg}$ ) and dysphagia progressively subsided over the next 12 hours, as assessed by the speech therapist.

\section{DISCUSSION}

The term "esophageal spasm" has historically emerged to clinically describe a motility disorder of the smooth muscle of the esophagus associated with chest pain and/or dysphagia. Over time, as gastroenterological diagnostic techniques have evolved, various pathological entities have been identified and "Distal Esophageal Spasm" (otherwise known as "DES") has become only one of the various forms of motility disorder such as achalasia, nutcracker esophagus, ineffective motility, hypertensive lower sphincter and jackhammer esophagus, which are diagnosed by manometry according to the Chicago Classification. Although esophageal manometry is the gold standard and EGDS is often normal, endoscopic evaluation can sometimes reveal esophageal spasm as a "corkscrew" appearance in the distal esophagus. Therefore, in the absence of other evident causes, according to the presence of dysphagia and the endoscopic appearance, we can legitimately advocate the diagnosis of esophageal spasm, even without manometric demonstration.

Specialist evaluation assessed the absence of deglutition disorders and dysphagia before the procedure and after resolution, excluding the possibility that the disease was previously present. According to our review of the literature, this represents the first report of esophageal spasm after electrical cardioversion. Tolone et al. described a case of jackhammer esophagus two weeks after catheter ablation for atrial fibrillation, assuming a radiofrequency-induced vagal nerve thermal injury as the provoking factor ${ }^{[4]}$. Khan and colleagues reported an episode of transient esophageal hypercontractility 4 weeks after lung transplantation, hypothesizing a vagotomy-related gastroesophageal reflux disease as the causative mechanism ${ }^{[5]}$. In consideration of the temporal correlation between the electrical cardioversion and the onset of esophageal spasm, we can hypothesize a cause-effect relationship. The well-known association between electric current and muscular contraction leads us to hypothesize that the defibrillating electrical shock somehow triggered an abnormal contraction of the esophageal smooth muscle. The involvement of the distal - retrocardiac - esophageal tract, directly crossed by the current between the antero-posterior plates, could be further suggestive of the cause-effect relationship, with this portion of the tract generally being the most commonly affected by esophageal spasm.

Despite the exceptional features of this case, we strongly support the clinical importance of this report. First, we suggest that such phenomena may be under-reported and that the exceptionality of our case was the severe presentation. Furthermore, an empirical approach through the administration of a spasmolytic therapy may be considered in the management of dysphagia after electrical cardioversion. Lastly, a cause-effect relationship may justify a different approach to electrical cardioversion in patients with known esophageal motility disease. In this case, antero-lateral pad positioning, when feasible, may prevent complications after the procedure.

\section{CONCLUSION}

We report a case of an esophageal spasm with complete dysphagia after electrical cardioversion. The strict temporal connection between the procedure and symptom onset and the involvement of the retrocardiac tract with sparing of the cranial portion suggest a cause-effect association between electrical cardioversion and esophageal spasm. 


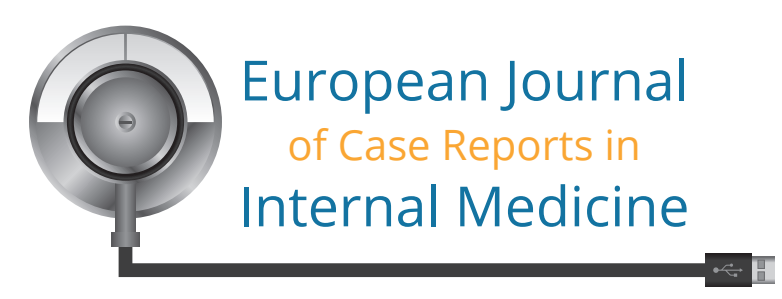

This assumption acquires greater value if we consider that the patient was not known for esophageal disease and/or symptoms before the procedure, a fact corroborated by the first dysphagia screening performed after stroke, which was normal. Therefore, clinicians should consider the possibility of an esophageal spasm when dysphagia develops after electrical cardioversion. This condition may be underreported and empirical treatment with a spasmolytic agent may rapidly resolve the clinical picture.

\section{REFERENCES}

1. Lufrano R, Heckman MG, Diehl N, DeVault KR, Achem SR. Nutcracker esophagus: demographic, clinical features, and esophageal tests in 115 patients. Dis Esophagus 2015:28(1):11-18.

2. Cattau EL Jr, Castell DO, Johnson DA, Spurling TJ, Hirszel R, Chobanian SJ, et al. Diltiazem therapy for symptoms associated with nutcracker esophagus. Am J Gastroenterol 1991;86:272-276.

3. Vanuytsel T, Bisschops R, Farre R, Pauwels A, Holvoet L, Arts J, et al. Botulinum toxin reduces dysphagia in patients with nonachalasia primary esophageal motility disorders. Clin Gastroenterol Hepatol 2013;11:1115-1121.

4. Tolone S, Savarino E, Docimo L. Radiofrequency catheter ablation for atrial fibrillation elicited "jackhammer esophagus": a new complication due to vagal nerve stimulation? J Neurogastroenterol Motil 2015;21:612-615.

5. Khan MQ, Nizami IY, Khan BJ, Al-Ashgar HI. Lung transplantation triggered "jackhammer esophagus": a case report and review of literature. J Neurogastroenterol Motil 2013;19(3):390-394. 
European Journal

of Case Reports in

Internal Medicine 